\title{
Modern technologies used in health crisis management during the Covid-19 pandemic
}

\author{
Nicu-Pantelimon Rotaru ${ }^{1, *}$, and Nicolae Taşcă ${ }^{2}$ \\ ${ }^{1}$ University of Petrosani, 332003 Petrosani, Romania \\ ${ }^{2}$ University of Petrosani, 332003 Petrosani, Romania
}

\begin{abstract}
During the last two years, the public health crisis caused by the SARS-CoV-2 pandemic has put constant pressure on social life, the functioning of institutions in general, and health services in particular. The rules of social distance or population mobility imposed by each state, determined by the level of spread of the virus, have shown how important various modern technologies are in facilitating relations between people or between them and state institutions or companies in which they work. Health services have directly benefited from the use of modern technology in health crisis management, but are also exposed to data security risks and the management of a large number of medical devices and consumables with limited use. In the context of the current crisis, the authors try to identify modern technologies and how they can facilitate the effective control of the spread of the pandemic, the exercise of a medical approach aimed at losing as few lives as possible, the discovery in record time of Covid-19 vaccines, the functioning of the economy and, very importantly, the maintenance of social relations in conditions of isolation. The authors' approach methodology was based on the analysis and interpretation of information presented by prestigious websites in the field of healthcare, scientific research or Information Technology.
\end{abstract}

\section{Introduction}

The context created by the Sars-CoV-2 (or Covid-19) pandemic has changed the perception of many concepts regarding public health at a very fast pace. The acceptance of modern technologies by decision-makers in the field of healthcare, in order to ensure efficient management of the crisis generated by the new coronavirus, was achieved in a relatively short time. In the medium and long term, there is still work to be done to reduce the anxiety and feeling of insecurity of patients and even specialists [1].

If before the beginning of this pandemic there was a long debate about the legislative, ethical, security aspects or the concrete ways of using Big Data in healthcare, telemedicine, etc., during the health crisis the attitude towards these concepts was reconfigured and began their implementation as useful tools for ensuring the best possible management of health services. Also, digital technologies have a crucial role in communication between front-line specialists, research centers in creating testing tools and vaccines, manufacturers in the

* Corresponding author: nicu.p.rotaru@gmail.com 
medical industry who develop equipment and devices for hospitals or decision makers involved in pandemic control, to find common solutions as soon as possible. It is a period marked by many innovations, of collective participation in finding solutions for controlling and combating the virus.

Healthcare management is facing an unprecedented period in the last century, but has the advantage of using technologies based on artificial intelligence (AI), telemedicine or other facilities of digital transformation in the field of healthcare. Even the top management of large multinational companies is starting to think about having their own health specialist within organizations.

In this paper, we will analyze how the current pandemic succeeded to condition the exercise of the managerial act by the use of new health technologies and what are the concrete benefits they bring in the field of healthcare, based on very recent scientific research.

\section{The current framework}

In the last 15 months, research efforts in various fields have intensified amid the spread of the SARS-CoV-2 virus. Under pressure from more than 120 million cases of infection, of which more than 2.5 million people died by the middle of March this year, researchers in various fields have made a continuous effort in scientific studies to overcome this period as much as possible, faster and with fewer casualties, so that the world's population can return to the normal pace of life.

A number of vaccines have been developed in record time, European Commission [2] granting conditional marketing authorizations for 3 of the 6 promising vaccines, namely those developed by BioNTech and Pfizer, Moderna and AstraZeneca. Of the contracted portfolio of 2.6 billion doses, the European Commission purchased 51.5 million doses by the end of February, with 29.2 million doses already administered to European Union citizens. Worldwide, Our World in Data, a statistical tool of the Oxford Martin School and the University of Oxford, respectively, mentions that 355 million doses were administered, 81.6 million people being completely vaccinated [2]. To understand the research effort to develop vaccines against the SARS-CoV-2 virus, a large study published in ScienceDirect [3] shows that the Covid-19 candidate vaccine project launched by the World Health Organization, in November last year, recorded a number of 48 candidates in the clinical evaluation, respectively 164 in different stages of the preclinical evaluation, 11 being in phase III studies.

Controlling the spread of the SARS-CoV-2 virus, the rapidity of the creation of vaccines to combat it, ensuring the proper functioning of intensive care units and, implicitly, the protection of first-line medical staff have become a priority for organizations in various fields, from medical or governmental to technologies, with a very important role for digital technologies [4]. The healthcare sector has been perhaps the most tried and tested area of the current pandemic, creating the possibility of digital transformation at an unprecedented rate for the healthcare industry. Timely decisions made by public health authorities under the pressure of the growing number of people infected with the SARS$\mathrm{CoV}-2$ virus have been facilitated by the collection of reliable data and evidence through the use of digital information and surveillance technologies. This pandemic has shown without a doubt that medical organizations can no longer respond to new challenges through traditional methods of data use, the large volume of data accumulated in a very short time from various sources, also referring to electronic health records (EHR), medical imaging, pharmaceutical research or medical equipment, showing us the importance of using Big Data in healthcare [5]. 
The current health crisis has shown the need to create the legislative and logistical framework for telemedicine / eHealth, effective in this context by long-distance relationships with patients, providing health services through high-speed telecommunications systems and software applications, protecting both physicians as well as patients from infection with the new coronavirus [6]. The gravity of the situation led to a cohesion of the factors involved in finding solutions to control and combat the SARS-CoV2 virus, open-source technologies offering the possibility to share information in real time and participate in decision-making through a collaborative attitude and unconditional access worldwide, thus solving the supply of test equipment, facial shields or ventilators. Anticipating the scale and speed of the vaccination process, digital technologies, such as open source, provide essential support for the planning, delivery, monitoring and management of the ongoing immunization programs [7]. The Coalition for Epidemic Preparedness Innovations (CEPI), Gavi, the Vaccine Alliance (previously Global Alliance for Vaccines and Immunization) and World Health Organization (WHO) manage a program to accelerate the development, production and equitable access to COVID-19 tests, treatments and vaccines [8]. Making a comparative analysis between the economic crisis of 2008, with the well-known effects of the years immediately following, with the current public health crisis, which inevitably affects the economic sector, we can see that if then researchers tried to make managers aware that information technology is the tool to be used with maximum benefits in decision-making and economic recovery, digital technologies are now the solution for efficient crisis management [9], [10].

\section{Applicability of modern technology during the health crisis}

The authors' approach is based on a structured analysis of relevant information presented in the papers of prestigious journals and conferences, official reports of organizations with a role in health crisis management or specialized sites such as: ScienceDirect, Taylor \& Francis, SpringerLink, Nature Medicine, European Journal of Public Health, NEJM Catalyst, ReliefWeb, Oxford Martin School, European Commission, WHO, World Bank or European Parliament. The specific keywords or phrases used to query the recently mentioned sources include: modern technologies, digital technologies, digital transformation, SARS-CoV2 / Covid-19, telemedicine, eHealth, healthcare devices, healthcare management, innovation, healthcare software / hardware, healthcare public, vaccine, health crisis, health financing or statistics Covid-19. Following the search stage, we selected the relevant articles for the proposed topic, following some guidelines: technological innovation, types of modern technologies, integration of digital technologies and technologies applied in the context of Covid-19.

The positive impact of technology on health is the digital transformation in this field, with some concrete examples in this regard: telemedicine, medical devices based on artificial intelligence (AI) or blockchain medical records, all substantially changing the dynamics between doctors, patients, providers, as well as how decisions about treatment and health outcomes are made [11]. In order to support the public health endangered by the current pandemic, the use of digital technologies is essential in decision-making by the management of the institutions responsible for controlling the new coronavirus, decisions that must be made under both the pressure of time and the loss of as few human lives as possible.

The European Commission has launched a call for SARS-CoV-2 in the H2020 Societal Challenge 1 to significantly increase the ability to respond to situations such as the one created by the current pandemic, promoting the acceptance of high-performance digital health technologies for prevention and of offering an optimized treatment. Thus, the European Commission funds under the program thirteen innovative solutions based on 
digital tools and artificial intelligence, these projects aiming at the protection of health professionals, the recognition and prevention of SARS-CoV-2 virus and the significant increase of the intensive care unit [12]. The projects submitted for funding are the following: ICU4Covid - Cyber-Physical Intensive Care Medical System for COVID-19; ENVISION - Intelligent plug-\&-play digital tool for real-time surveillance of COVID-19 patients \& smart decision-making in ICUs; IRIS-COV - Market Release of a Portable Device for COVID-19 at the Point-of-Care; a Global Diagnostics Approach; COVIRNA A diagnostic test to improve surveillance and care of COVID-19 patients; CorDial-S Portable and fast surface plasmon resonance point-of-care test for COVID-19; VASCOVID - Portable platform for the assessment of microvascular health in covid-19 patients at the intensive care; PyXy.AI - Telehealth-ready AI-powered multi-parametric system for surveillance of COVID-19 and cardio-pulmonary chronic patients; ESSENCE - Empathic platform to personally monitor, stimulate, enrich, and assist elders and children in their environment; icovid - AI-based chest CT analysis enabling rapid COVID diagnosis and prognosis; PORSAV - Controlling viral aerosols in COVID-19 and beyond; CleanAir - Lab to Fab development of air decontamination system for protecting health practitioners against COVID-19; INNO4COV-19 - Boosting Innovation for COVID-19 Diagnostic, Prevention and Surveillance; COVID-X - COVID eXponential Programme.

In the face of a threat that appeared very quickly and with serious effects for the population, the European Union considered it essential to finance projects involving digital technologies, high quality data or artificial intelligence, in response to this pandemic. In April last year, the European Parliamentary Research Service presented a detailed analysis of ten modern technologies used in the management of the health crisis caused by the new coronavirus, as follows: artificial intelligence, blockchain applications, open-source technologies, telehealth technologies, three-dimensional technologies, gene-editing technologies, nanotechnologies, synthetic biology, drones and robots. If artificial intelligence, drones and robots are used to apply restrictive measures and identify the virus, scientists are also studying synthetic biology, gene-editing technologies and nanotechnologies for vaccine discovery and testing, and of diagnoses and treatments. Maintaining medical supply chains, managing insurance payments and tracking contagion can be accomplished using blockchain applications. The efforts of hospitals and authorities to optimize the supply of the necessary medical equipment and ensure the necessary medical hardware, such as face masks, fans and respirators, are provided with threedimensional technologies and open-source technologies. Telehealth technologies act as a tool to filter people with moderate conditions that can stay at home, from severe ones that are directed to the hospital, thus controlling the spread of the virus and maintaining the capacity of hospitals, especially intensive care units [13].

WHO has identified 12 functions of digital health technologies, focusing on providing relevant information on health and disease, supporting professionals in the field of diagnosis and treatment, providing verifiable recorded data, real-time access to managers from different levels to operational and strategic information regarding the various necessary resources (medicines, finance, logistics, human) [14]. Innovation appears to be an absolute necessity during infectious hazards with a potential for global expansion such as the SARS-CoV-2 pandemic. Each country needs to develop its capacity to prepare for such situations, so the need to discover innovative methods and technologies becomes imperative. The following diagram shows how digital technologies are capitalized in response to the fight against the new coronavirus, with advantages and limitations alike, being a systemic approach with a role in establishing SARS-CoV-2 control strategies based on digital technologies [10].

Big Data has created a completely different perspective on the use of data (management, analysis and capitalization) in all industries, but especially in the field of healthcare. In the 
area of healthcare, Big Data refers to the large amounts of information collected from patients' medical records through digital technologies, impossible to achieve using traditional technologies, with the aim of better hospital management. Considering the security risks and ethical issues, Big Data for healthcare has many benefits that can lead to saving lives, preventing even future epidemics, and from a financial perspective leads to high-cost reductions.

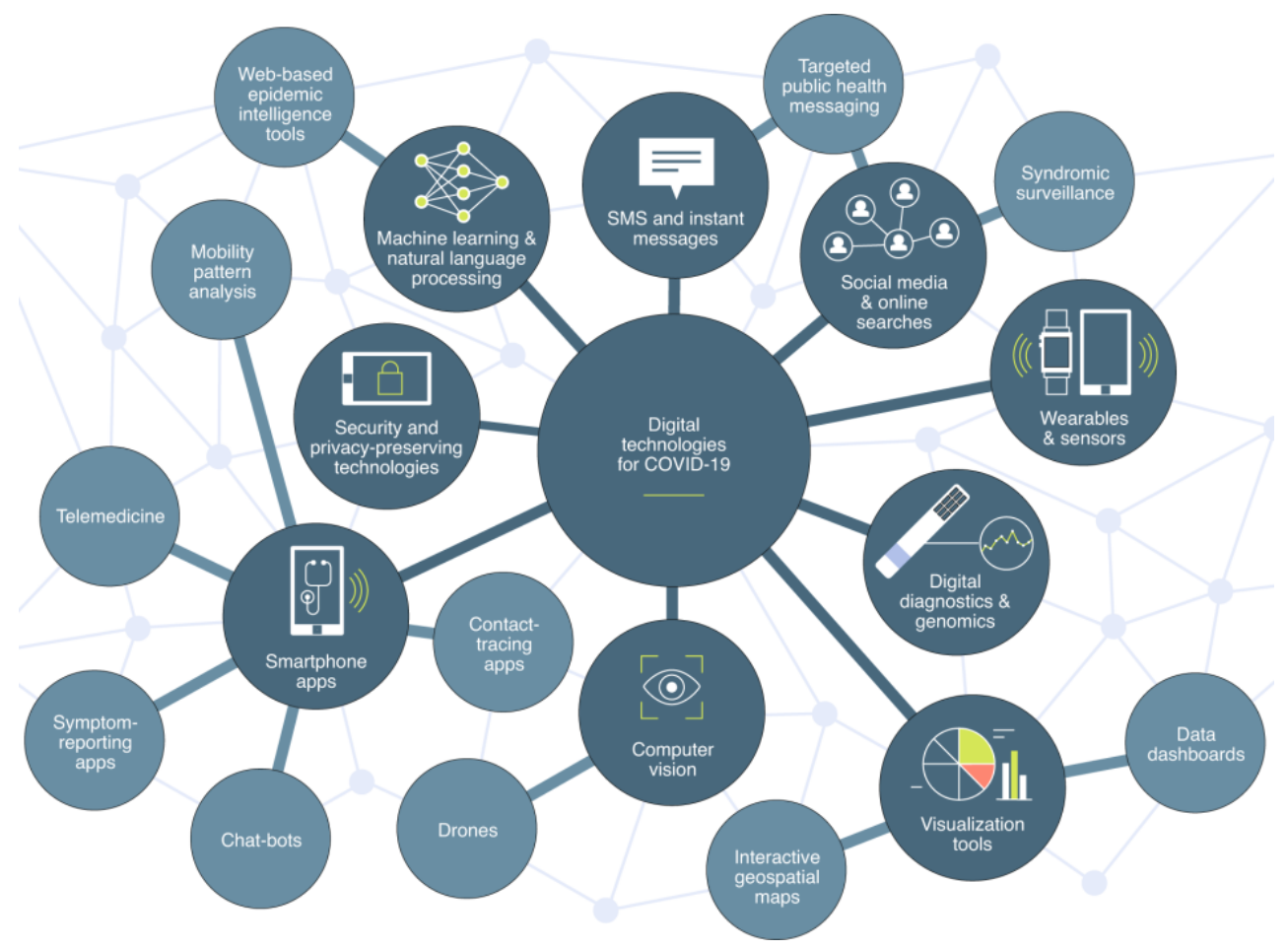

Fig.1 The interconnected digital technologies used in the public-health response to SARSCoV-2 [10]

Digital technologies (Figure 1) facilitate the collection of a huge volume of data, generate reports that are transformed into valuable information, and then used to provide quality health care. Prevention of public health problems and both intervention with appropriate methods and treatments is the purpose of analyzing data on healthcare.

We highlight 18 applications that Big Data can have in health care, the results being the improvement of medical processes and patient care: improved patients' predictions, improved supply-chain, medical imaging, real-time alerting, augment cancer treatments, Electronic Health Records (EHRs), telemedicine, improved strategic planning, prevent opioid abuse, predictive analytics, reducing fraud, enhancing patient engagement, developing new therapies, optimizing ER admissions, smart staffing, learning and development, risk and disease management, self-harm prevention [15]. Big Data applied on the hospital dashboard provides the manager with information about each division in a single point, as well as an overview of the health facility (Figure 2); respectively the dynamic patient dashboard, a tool designed to increase service levels and communication between departments for precision treatment during Covid-19 pandemic (Figure 3). 


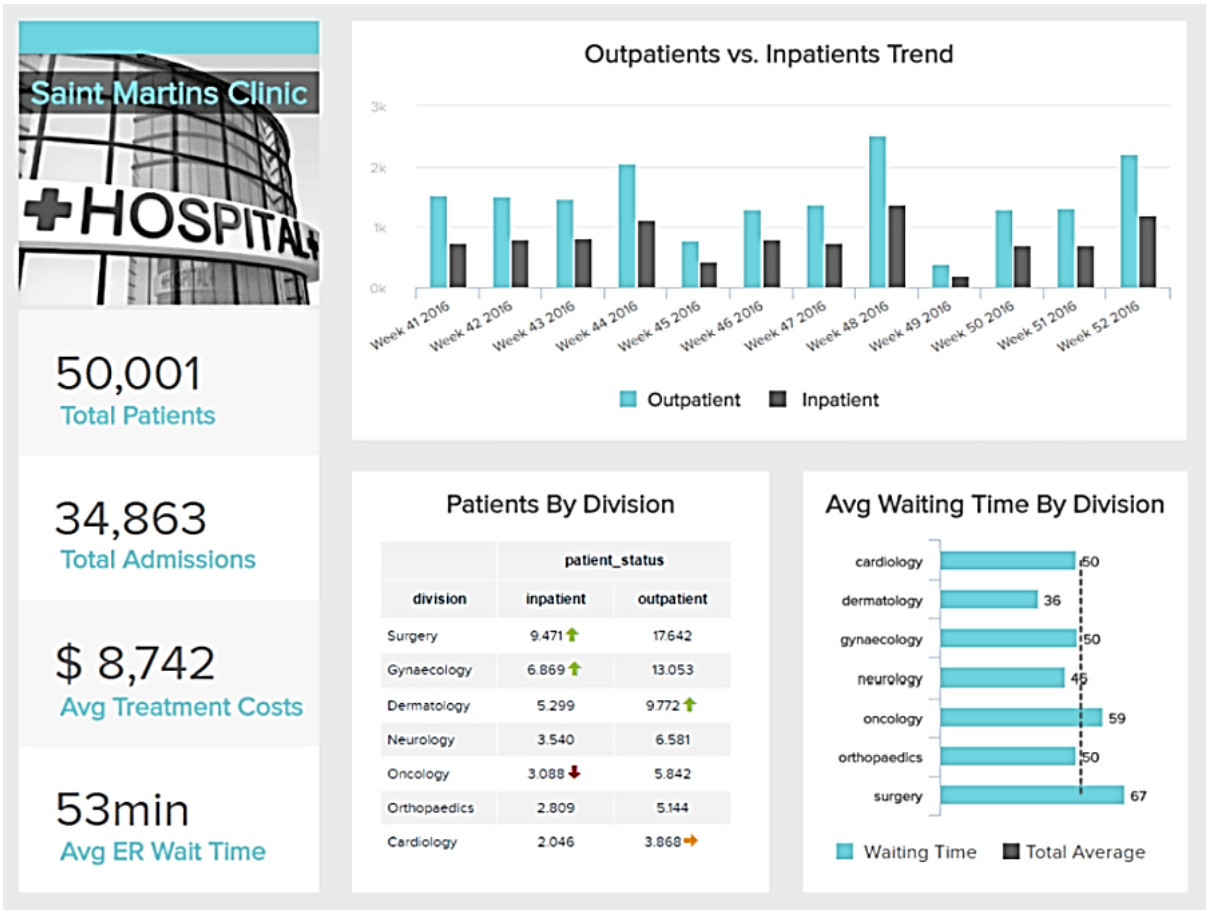

Fig.2. Hospital dashboard, a useful tool for the manager [15].

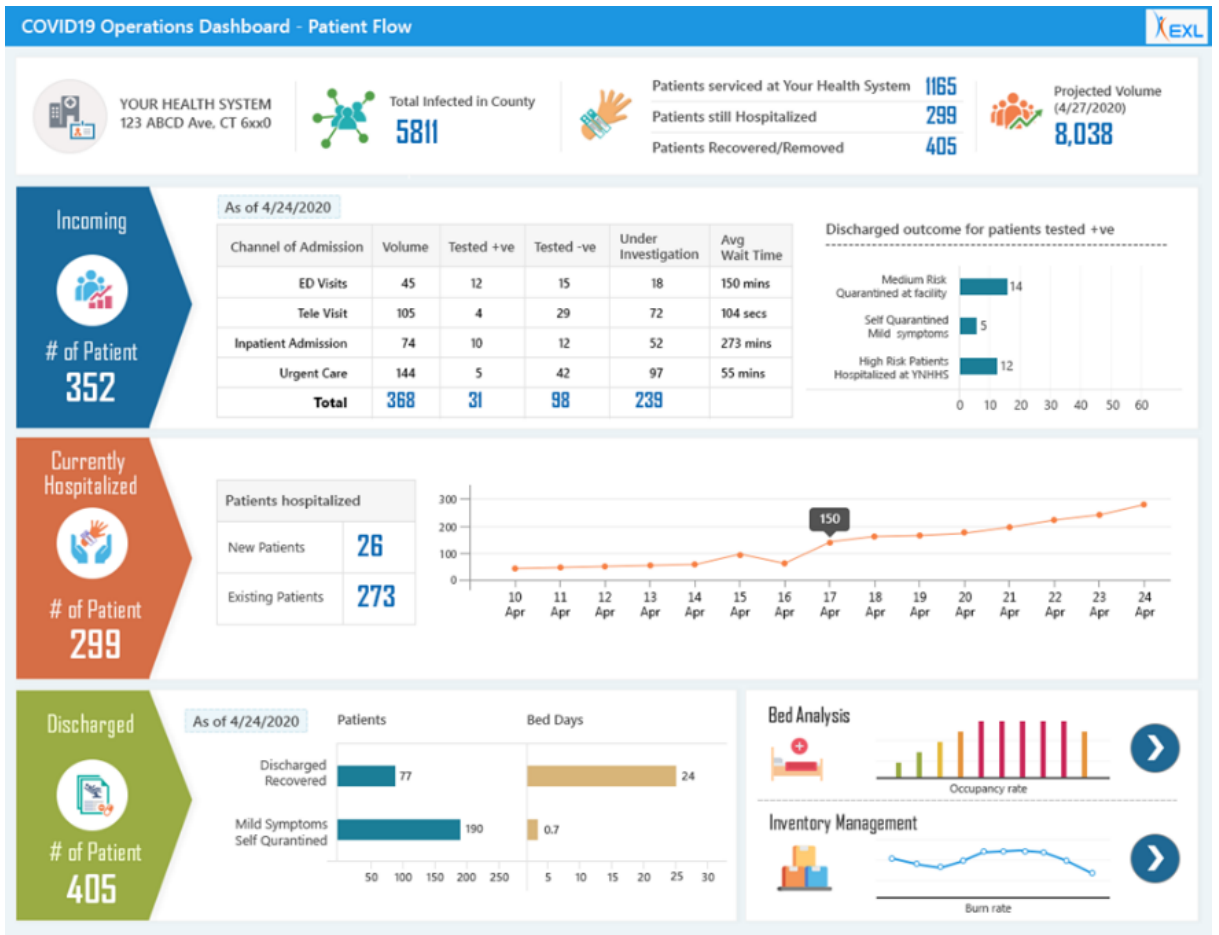

Fig.3. Patient dashboard, a useful tool for the departments [16]. 
Based on other algorithms for analyzing health records and monitoring patient contact history in order to identify patterns of virus spread, Big Data has the potential to detect outbreaks by gathering information from a variety of sources. Based on these applications, both current and predicted future outbreaks can be identified, monitoring the contacts. Prevention of future Covid-19 alarms can also be achieved with the artificial intelligence technique called Natural Language Processing (NLP), based on constant interactions in the form of text and speech, through this technique can be assigned different meaning to human communication [17].

The use of Big Data in the field of health care has both advantages and disadvantages, so the large-scale insertion of this technology must be done taking into account a multitude of factors. Advantages of Big Data in health care: access to real-time information, facilitation of rapid diagnoses and effective and quality treatments, identification of risk factors to prevent disease, making medical decisions in a more informed way, identification and overcoming the operational problems of the organization, applying for predictive analysis, reporting fraud, etc. Big Data in health care involves ethical and legal issues, including confidentiality, public transparency, personal autonomy, data protection, sharing, necessary infrastructure, negative effects on patients' rights and freedoms [18], [19].

Applications of Big Data in SARS-CoV-2 pandemic: identifying possible positive cases, analyzing the risk based on the history of patient movements, suggesting the need for medical assistance in identifying suspicious cases, recognizing an early stage of a patient infected with Covid-19 virus, identifying and analyzing disease dynamics, monitoring the population and supporting health management during the lockdown, it facilitates the rapid obtaining of treatments against the virus [20]. Big Data has supported the generation of computational models to predict the spread of the virus based on the use of specific analytical techniques, such as behavioral models (SIR model - Susceptible, Infectious, and Recovered, has been supplemented with new behaviors, such as Exposed, Hospitalized, and Funeral); dynamic cascade models (Independent Cascade Model and Linear Threshold Model); Compartmental models (differentiating between common cold (SIS) and Corona (SEIR), extended with host with no symptoms (SEIaR) [20, 21].

Telehealth or telemedicine technologies, although we must emphasize that they are not entirely identical terms, refer to the diagnosis of patients by doctors remotely, through twoway audio-video communication systems. Interaction is performed via webcam devices, chatbots and automated algorithms. Remote healthcare provides several advantages in the current situation, as follows: hospitals are available for confirmed cases, the contagion rate is reduced, and the doctor can treat several patients at the same time. Currently, online checks are performed to identify symptoms of Covid-19 in patients, as well as to obtain a detailed history of travel or exposure [13].

The process of telemedicine application in the provision of clinical services during the Covid-19 pandemic is achieved through synchronous and asynchronous e-health platforms, as follows [6]:

- Mobile integrated health care programs or community paramedicine - provides patients with superior medical care at home through digital surveillance by doctors. This type of intervention is performed through emergency channels, such as 911 or 112, but eliminating the transport to the emergency department.

- Mobile Health (mHealth) application - through a software application installed on mobile devices or portable devices that have access to the Internet, the doctor manages, on the one hand, healthcare operations, analyzes data and contributes to improving the patient experience, and on the other hand has the possibility of disseminating information with other specialists. 
- Artificial Intelligence (AI) and machine learning decision making application - the implementation of AI Chatbot provides both information about Covid-19 and suggestions for preliminary testing of symptomatic patients and administration of treatment. The high volume of patients overloading healthcare channels can also be reduced. The identification of Covid-19 outbreaks can also be predicted, and AI applications facilitate the minimization or acceleration of diagnosis and monitoring processes. Through machine learning, algorithms can learn and develop the accuracy of virus identification.

- Robotic technologies - Robotic technologies aim to provide support to people with disabilities or volunteers who assist patients positively identified with SARS-CoV-2, for rehabilitation, nursing and medical robotics. These technologies have been used in China to assist patients in isolation or quarantine areas. Robots have the ability to interpret patients' facial expressions, voice recognition and equipment management.

- Social networking applications (Facebook, Skype, WhatsApp, FaceTime, etc.) - these applications demonstrate their usefulness in conditions of self-quarantine and isolation, having the ability to compensate for the lack of face-to-face interaction, especially for people at risk of depression.

- Contact tracing apps category - these applications are used worldwide to manage the spread of the new coronavirus, with some concrete examples: TraceTogether, developed by the Singapore government to monitor infected people and possible contacts; the Israeli government has legislated the possibility of monitoring the mobile devices of people suspected of being infected; Taiwanese health authorities have access to monitoring the mobile devices of quarantined persons.

- Medical application - addressed especially to patients in underdeveloped areas, with limited access to health care, isolated patients in case of natural disasters or in emergencies when demand is high, facilitating both synchronous and asynchronous health care services (applications of primary care: K Health, Teladoc și Doctor on Demand).

- Health and fitness applications- in conditions of quarantine and social isolation, these applications have the role of stimulating active behavior, to protect the physical and mental health of the population (e.g.: MyFitnessPal provides a diet plan and calorie counter).

Telemedicine / telehealth offers advantages such as: access to medical records in isolated areas, such as rural areas or disaster areas; low costs for both patients and providers; control of the spread of diseases, such as Covid-19; comfortable; easy monitoring; doctors may have more patients; gain time for both parties, specialists and patients. There are undoubtedly disadvantages in the use of telemedicine / telemedicine technologies: requires internet access, isolated areas rarely having access; limitations in patient evaluation; possession of smart devices; legislative limitations; protection of patient data [22].

\section{Discussion}

Big Data should not be considered the life-saving solution for health systems in the fight against Covid-19, but it must be considered the huge capacity of data provided in real time, as a result of technological conquests and access of the population to personal smart devices have made it possible. approximately $90 \%$ of the global volume of data to be collected only in 2017, with the capacity to multiply 40 times by 2020 . By connecting this huge volume of data in real time, a pandemic management can be achieved through Geographical Information Systems (GIS), facilitating the identification and monitoring of outbreaks. 
The concrete structure of Big Data using in the management of the current health crisis is presented as follows:

- collecting data from Big Data sources (Internet of Things - IoT, mobile data, social networks, navigation and search engine, large-scale gene Bank);

- data analysis (visual analysis - graph database decision, GIS; deep learning - neuronal network, NLP; predictive analysis - correlation analysis, predictive modeling analysis);

- problem solving (incidents warning based on network information, forecast analysis of incidents trend, real-time disease surveillance system, analysis of patient infection path, close contacts search analysis, real-time query and report of diseases around users, home quarantine declaration and statistics, dispelling rumors, analysis and appease public panic, emergency supplies and equipment tracking, host and virus infection pattern analysis, infection disease prediction model, organize resumption of work and daily operation prevention and control, incidents impact and policy evaluation and analysis);

- epidemic prevention mechanism (prevention of major public health incidents, response to major public health incidents, recovery of major public health incidents)

- related subjects (government agency, enterprises and institutions, medical institutions, users) [23].

The Centers for Disease Control and Prevention (CDC) noted a 50\% increase in medical visits through telehealth technologies in the first quarter of 2020 compared to the same period last year, with the specification that requests were for issues other than Covid-19 [24].

The life cycle of the telemedicine process involves the following steps: 1 . patient reads information on telemedicine on hospital platform; 2. patient consents and registers by entering full name, phone number and national ID; 3. patient downloads telemedicine software such as Zoom, Skype etc.; 4. patient logins and makes appointment: 5. data and time of reservation is confirmed by the attending medical staff and send to the patient; 6 . patient and physician join the consultation based on the meeting ID at the appointed time; 7. physician starts the assessment diagnosis, and treatment of patient; 8 . future follow-up appointment is scheduled for monitoring patient. Legislative details on consent differ from country to country, with some accepting only verbal agreement while others involve the General Data Protection Regulation (GDPR), and the process can be more difficult [25].

\section{Conclusion}

The authors consider that the current health crisis caused by the Sars-CoV-2 (or Covid-19) pandemic is both a huge challenge for health systems around the world, but also an opportunity for the integration of digital technologies in healthcare. Limiting the loss of human life through a combined effort of researchers, organizations, authorities and, implicitly, the population, demonstrate the extremely important role of using modern technologies for health in order to: obtain real-time information, record production of lifesaving medical equipment in record time, human resources, front-line personnel protection, obtaining Covid-19 vaccines in an unprecedented time, controlling the spread of the virus and communication in order to ensure effective management of the health crisis.

Funding for projects involving innovative health technologies has been accelerated, with organizations aware that overcoming the crisis depends on using the tools resulting from these innovative projects. Telemedicine / eHealth has proven effective during this period, eliminating the risk of infection with the new coronavirus both for patients, but especially for medical staff, helping to optimize the capacity of intensive care units. In our opinion, Big Data began to inevitably and irreversibly transform healthcare during this pandemic. Improving patient care and value-based medical units will build on the solutions 
offered by Big Data in healthcare. The equitable distribution of vaccines globally with the help of a unique program is an approach to the management of the largest vaccination campaign of all time, unequivocally based on the use of digital technologies for healthcare.

Analyzing both the positives and the negatives of the large-scale integration of digital healthcare technologies, the authors predict that it is a long process, which involves: harmonizing many factors globally, creating a legislative framework to ensure confidentiality and respect for ethical issues; the implementation of a large-scale technological infrastructure to cope with such a large volume of data, but above all to ensure data security; overcoming barriers in terms of 5G mobile technology for instantaneous healthcare; and, most importantly, investment in high quality human resources (IT experts, data architects and big data engineers). Particular attention must be paid to maintaining the accuracy of particularly complex social realities, any error in the use of information can have negative effects on the rights or health of citizens.

Healthcare management at various levels will be one of the major beneficiaries of the digital transformation in health, having real-time access to any information about the organization they lead with a simple dashboard or instantly generating a report. Doctors will diagnose and treat patients much safer and faster, based on data shared with colleagues around the globe, exchanging best practices and know-how being easier than ever. Last but not least, the patient is the great beneficiary of this transformation, his access to healthcare services being much improved, both at home and when he travels, considering the access to the electronic health record.

Accepting absolutely all the negative aspects of the Covid-19 pandemic, the authors emphasize the idea that it offered the opportunity to use modern technologies in support of public health, representing the key moment in what we know as the digital transformation in healthcare. The control of the spread of this virus and the limitation of the number of loss of human lives were possible with the help of the technologies described in the paper. Global resilience is undoubtedly be conditioned by the use of the mentioned technologies.

\section{References}

1. Safi S., Thiessen T., Schmailzl K.J.G., JMIR Res Protocols 7, 12 (2018)

2. European Commission. Safe COVID-19 vaccines for Europeans, https://ec.europa.eu/info/livework-travel-eu/coronavirus-response/safe-covid-19-vaccines-europeans_en (2021)

3. H. Ritchie, E. Ortiz-Ospina, D. Beltekian, E. Mathieu, J. Hasell, B. Macdonald, C. Giattino, C. Appel, M. Roser. Statistics and Research. Coronavirus (COVID-19) Vaccinations, https://ourworldindata.org/covid-vaccinations (Our World in Data, 2021)

4. S. Chakraborty, V. Mallajosyula, C. M. Tato, G. S. Tan, T. T. Wang, Advanced Drug Delivery Reviews 172, 314-338 (2021)

5. NEIJM Catalyst. Healthcare Big Data and the Promise of Value-Based Care, https://catalyst.nejm.org/doi/full/10.1056/CAT.18.0290 (2018)

6. Bokolo, A.J., Health Technol. 11, 359-366 (2021)

7. B. Guermazi, Digital technologies and vaccine deployment: Opportunities and challenges https://blogs.worldbank.org/digital-development/digital-technologies-and-vaccine-deploymentopportunities-and-challenges (2021)

8. World Health Organization, COVAX Working for global equitable access to COVID-19 vaccines, https://www.who.int/initiatives/act-accelerator/covax (2021)

9. E. Edelhauser, A. Ionică, Comput. Sci. Inf. Syst. 11(2), 809-823 (2014)

10. Budd, J., Miller, B.S., Manning, E.M. et al., Nat Med 26, 1183-1192 (2020)

11. M. Reddy, Digital Transformation in Healthcare in 2021: 7 Key Trends, https://www.digitalauthority.me/resources/state-of-digital-transformation-healthcare/ (2021) 
12. European Commission, Digital health technologies addressing the pandemic, https://ec.europa.eu/digital-single-market/en/digital-health-technologies-addressing-pandemic (2021)

13. European Parliamentary Research Service, Ten technologies to fight coronavirus, https://www.europarl.europa.eu/RegData/etudes/IDAN/2020/641543/EPRS_IDA(2020)641543_ EN.pdf (2020)

14. M. Mitchell, L. Kan, Health Systems \& Reform 5(2), 113-120 (2019)

15. S. Durcevic, 18 Examples of Big Data Analytics in Healthcare that Can Save People, in Business Intelligence, https://www.datapine.com/blog/big-data-examples-in-healthcare/ (2020)

16. EXL Service, Covid-19 Healthcare Providers Operations Dashboard, https://info1.exlservice.com/hubfs/EXL-Media-Library/Solution-Sheet/EXL-SS-COVID-19Operations-Dashboard.pdf (2021)

17. Z. Siddiqui, F. Rathinam, Big data in the time of a pandemic. News and Press Release https://reliefweb.int/report/world/big-data-time-pandemic (2021)

18. Pastorino R., De Vito C., Migliara G., Glocker K., Binenbaum I., Ricciardi W., Boccia S., European Journal of Public Health 29, 23-27 (2019)

19. Fatt Q.K., Ramadas A., J Healthcare Communications 3, 21 (2018)

20. Haleem A., Javaid M., Khan I.H., Vaishya R., Indian J Orthopaedics, 54 (4), 526-528 (2020)

21. Villanustre F., Chala A., Dev R. et al., J Big Data 8, 33 (2021)

22. Moneypenny M., The Enormous List of Telehealth Pros and Cons, https://etactics.com/blog/telehealth-pros-and-cons (2020)

23. Jia Q., Guo Y., Wang G., Barnes SJ. Int J Environ Res Public Health 17, 17 (2020)

24. Koonin L.M., Hoots B., Tsang C.A., et al., MMWR Morb Mortal Wkly Rep 69(43), 1595-1599, http://dx.doi.org/10.15585/mmwr.mm6943a3 (CDC, 2020)

25. Bokolo A. Jnr., J Med Syst 44, 132 (2020) 